\title{
Gaussian process as complement to test functions for surrogate modeling
}

\author{
Raphael T. Haftka ${ }^{1}$ (1) $\cdot$ Chanyoung Park ${ }^{1}$
}

Received: 26 March 2019 / Revised: 21 August 2019 / Accepted: 20 October 2019 / Published online: 8 February 2020

(C) Springer-Verlag GmbH Germany, part of Springer Nature 2020

\begin{abstract}
It is common for papers on surrogate fitting to select test functions for testing algorithms. This raises the issue of how well the algorithms generalize to other functions. This editorial proposes as a possible complement to use a Gaussian process (GP) for generating test functions. The GP is defined by spatial decay rates of correlation between function values at different locations. The selection of these decay rates is tantamount to selection of the wave lengths of the functions that can be approximated by the surrogate. A possible complement to a given test function is a GP with similar decay rates to those of the test function, which would generate random functions with similar waviness. Two approaches are considered. In the simpler one, the GP is used to generate random test functions at a predetermined set of points. The more elaborate scheme targets situations where some samples are already available, and the GP is restricted to generate random functions that interpolate the samples. The process is illustrated for a one-dimensional case, where we seek to measure the correlation between the $95 \%$ confidence interval of the GP fit and the actual error. It is hoped that the editorial will stimulate discussion of the problem of algorithms customized to particular test functions and other options besides the one proposed here.
\end{abstract}

\section{Introduction}

It is common for papers on surrogate fitting to select test functions, such as the Branin-Hoo function or the Hartman set of functions for demonstrating the effectiveness of the algorithms. On the one hand, it is good that particular test functions are popular, because that permits new algorithms to be easily compared with previously published algorithms. On the other hand, one wonders whether algorithms get customized to a particular set of test functions and do not generalize well to other functions. One objective of this editorial is to propose a complement to standard test functions in the form of random functions generated from a Gaussian processes (GP). Another objective is to solicit comments and discussion on the issue of selecting test functions for testing algorithms.

Responsible Editor: Vassili Toropov

Electronic supplementary material The online version of this article (https://doi.org/10.1007/s00158-019-02441-1) contains supplementary material, which is available to authorized users.

Raphael T. Haftka

haftka@ufl.edu

1 University of Florida, Gainesville, FL, USA
A GP is defined by a mean function that defines its trend, and a correlation function that defines the correlation between function values based on their location in space. The correlation decays with distance, and the decay rates (often called hyper-parameters of the GP) define the wave lengths of the random functions that can be generated from the GP. The selection of the decay rates by the tester explicitly defines how wavy the target function for the surrogate fitting algorithm can be. A natural complement to a given test function is a GP with decay rates that are the same or similar to the test function. It will generate random functions with similar waviness to the test functions, and some of them will not share idiosyncrasies of the given test function. Similarly, the mean function can be selected to be similar to the trend of the test function.

Once a GP is used to generate random test functions, the testing can be repeated multiple times. Then the performance of an algorithm is expressed as a distribution rather than as a deterministic value.

Two approaches for using the GP are proposed here. The simpler approach is to generate random test functions at a predetermined set of points, such as a dense grid. This simpler approach is useful where we can determine ahead of time where we need to evaluate the function. The second approach caters to adaptive sampling algorithms, where sampling 
locations are determined based on values at previous samples. Then we need to generate random functions that interpolate the previously available samples. This also caters to algorithms that are motivated by a particular application. Often an algorithm is tested for a particular application by fitting a surrogate to given data and then checking the error at validation points. It is then difficult to tell whether good or bad performance is a matter of chance or inherent. Therefore, it makes sense to use the GP to generate random functions that all pass through the samples. Finally, this approach is useful for generalizing a given test function that may have a possibly undesired idiosyncrasy. For example, the Branin-Hoo function is a favorite test function for surrogate-based optimization algorithms. However, it has three local optima with the same function value, which make them all global optima. This property may make it easier to optimize. By using a GP constrained to pass through a large sample of points from the Branin-Hoo function, we can generate very similar functions that have different values at the three local optima.

In this editorial, we illustrate the process for a zero-mean GP, which is the simplest case. However, comments in the Matlab codes in the appendices indicate how to add a mean function if desired.

Section 2 describes the procedure of generating a random function from a given GP. Section 3 describes the procedure of generating random functions that all interpolate previously available samples. Both are standard procedures that are summarized here for the convenience of the reader. Section 4 illustrates the process for a one-dimensional case, where we seek to measure the correlation between the $95 \%$ confidence internal of the GP surrogate fit and the actual error. Section 5 offers concluding remarks.

\section{Gaussian process for generating random functions}

Consider the problem of fitting a surrogate to a function $\mathrm{f}(\mathbf{x})$ of an n-dimensional vector $\mathbf{x}$ in a domain $\mathrm{S}$. This is done by sampling the function at a set $\boldsymbol{x}^{d}$ of $k$ data points $\mathbf{x}_{1}^{d}, \ldots, \mathbf{x}_{k}^{d}$ to obtain a vector $\boldsymbol{f}^{\boldsymbol{l}}$ of function values. While algorithms are often invented for a particular $y$ (e.g., the aerodynamic efficiency of a wing), they are often tested on a particular set of test functions. It is common to randomize $\mathbf{x}^{\mathbf{d}}$, for example by using Latin hypercube sampling (LHS), but the set of test functions is deterministic. Here it is proposed to randomize also the test functions by sampling them from a Gaussian process (GP).

A GP is a generalization to a random function of a normally distributed random vector. To generate the vector of function values $\mathbf{f}^{\mathbf{d}}$ as a normally distributed vector, we need to specify a vector of mean values $\mu^{\mathbf{d}}$ and a covariance matrix $\Sigma^{\mathrm{dd}}$. If the function values are generated by a GP, we assume that the correlation between function values is high if the distance between the $x$ locations is small. That is, the covariance matrix is determined by a variance value $\sigma^{2}$ and a correlation that is determined by the distance between the points, with strong correlation for nearby points and weak correlation for points that are far from each other.

For example, with a Gaussian correlation function (note that a GP can have other correlation functions), the correlation between function values at point $\mathbf{x}$ and point $\mathbf{s}$ is given as

$C(f(\mathbf{x}), f(\mathbf{s}))=\prod_{i=1}^{n} \exp \left(-\theta_{i}\left(x_{i}-S_{i}\right)^{2}\right)$

where the vector $\theta$ determines how fast the correlation decays between points. The covariance matrix corresponding to the GP is the variance multiplying the matrix of correlations. That is

$$
\Sigma^{d d}=\sigma^{2}\left[\begin{array}{ccc}
C\left(x_{1}^{d}, x_{1}^{d}\right) & \cdots & C\left(x_{1}^{d}, x_{k}^{d}\right) \\
& \ddots & \vdots \\
S y m & & C\left(x_{k}^{d}, x_{k}^{d}\right)
\end{array}\right]_{(k \times k)}
$$

Note that the matrix is symmetric and the diagonal elements of the correlation matrix are equal to 1 because the correlation of the function value at a point with itself is 1 . The GP also needs the mean $\mu(\mathbf{x})$. However, since we want to avoid the selection of particular functions, here it is proposed to set $\mu(\mathbf{x})=0$, so the only parameters defining the GP are $\sigma^{2}$ and the decay rates $\theta_{\mathrm{i}}$. For example, Fig. 1 shows two random functions generated from a one-dimensional GP with

$$
\sigma^{2}=0.01, C(x, s)=\exp \left(-5(x-s)^{2}\right)
$$

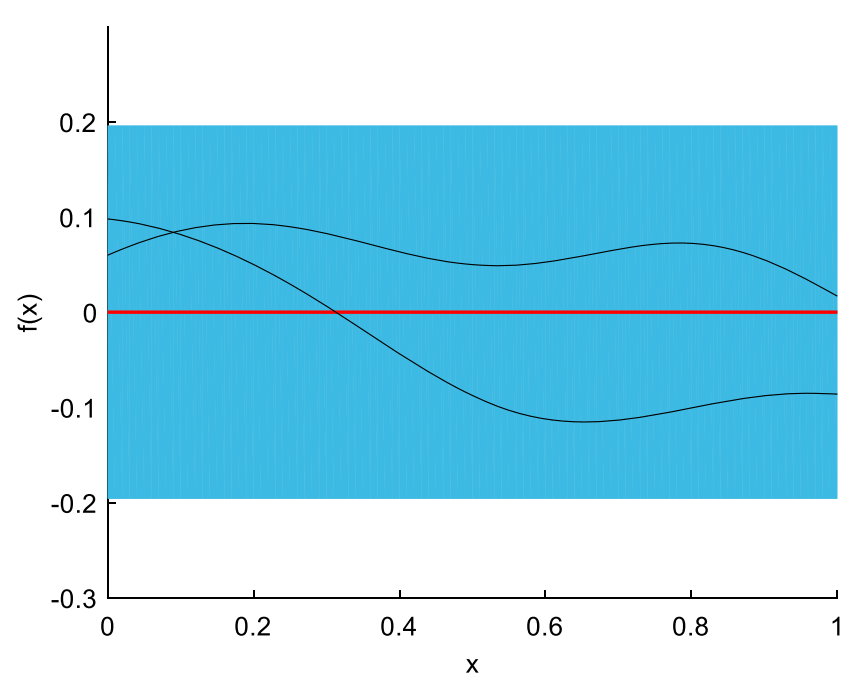

Fig. 1 Two random functions (blue lines) generated from the GP defined with (3), with the mean shown in red and the shaded region corresponding to the $95 \%$ confidence interval 
The Matlab code given in Appendix 1 generates random functions from this GP at 101 equally spaced points. If in order to test an algorithm, we need the test function at predetermined locations, the code given in Appendix 1, generalized to $n$ dimensions would be all we need.

\section{GP constrained to interpolate previous samples}

We now want to limit ourselves to random functions that interpolate the data and sample them at another set of q points $\mathbf{x}^{\mathrm{s}}$. This would be of use when we do not know ahead of time all the points where we need the random function so that the data comes from previous samples, or when data comes from a particular application. We create the covariance matrix for the combined set of points (data and new samples) and partition it as

$\Sigma=\left[\begin{array}{ll}\Sigma^{s s} & \Sigma^{s d} \\ \Sigma^{d s} & \Sigma^{d d}\end{array}\right]$

where

$$
\begin{aligned}
\Sigma^{s s} & =\left[\begin{array}{lll}
C\left(x_{1}^{s}, x_{1}^{s}\right) & \cdots & C\left(x_{1}^{s}, x_{q}^{s}\right) \\
& \ddots & \vdots \\
& & C\left(x_{q}^{s}, x_{q}^{s}\right)
\end{array}\right]_{(q \times q)} \\
\Sigma^{s d} & =\left[\begin{array}{ccc}
C\left(x_{1}^{s}, x_{1}^{d}\right) & \cdots & C\left(x_{1}^{s}, x_{k}^{d}\right) \\
& \ddots & \vdots \\
& & C\left(x_{q}^{s}, x_{k}^{d}\right)
\end{array}\right]_{(q \times k)}=\left(\Sigma^{d s}\right)^{T}
\end{aligned}
$$

We denote a random function that interpolates the given data by $\mathrm{y}(\mathbf{x})$. To generate random samples of $\mathrm{y}\left(\mathbf{x}^{\mathbf{s}}\right)$, we need the conditional distribution given the data. This requires updating the mean as

$\mu_{\text {upd }}^{s}=\mu^{s}+\Sigma^{s d}\left(\Sigma^{d d}\right)^{-1}\left(\mathbf{f}^{d}-\mu^{d}\right)$

If the previous samples are from the same zero-mean GP, then $\mu^{d}=0$. Similarly, the covariance matrix of the sampling points is updated as

$\Sigma_{u p d}^{s s}=\Sigma^{s s}-\Sigma^{s d}\left(\Sigma^{d d}\right)^{-1} \Sigma^{d s}$

A Python code for generating a two-dimensional GP with non-zero mean is available in the supplementary material.

\section{Example: correlation between confidence interval and error}

At each one of the sample points $\mathbf{x}^{\mathbf{s}}$, the prediction is a normal distribution with the updated mean and with a variance equal to the diagonal element of $\Sigma_{\text {upd }}^{s s}$. This property is widely used as a stand-in for the error in the surrogate in algorithms for adaptive sampling and algorithms for estimating the probability of failure. A recent paper by Zhang et al. (2019) noted that for sparse samples, the correlation between the confidence interval and the actual error was rather low for some selected test functions including the Hartman 6 function.

The results by Zhang et al. motivated doing the example presented here to explore the correlation for the onedimensional case of fitting a GP surrogate to two points in 0 $\leq x \leq 1$. To simplify the example, we assume that we select two sample points by optimal LHS, which would put them at $\mathrm{x}=$ 0.25 and $x=0.75$. We simplify further by considering here the ideal case where $\theta$ is known rather than obtained from maximum likelihood.

Figure 2 shows one example for $\sigma^{2}=1, \theta=5$. For this mild waviness, two points are not too sparse. The initial random function had $f(0.25)=-0.9007, f(0.75)=0.8283$. The GP fit to that data is shown by the red curve (mean) and one random function is shown with the dashed line. For this random function, the correlation between error and confidence interval (calculated at 50 equally spaced points) is 0.8554 .

Figure 3 shows another case where the initial GP generated $f(0.25)=-0.0649, f(0.75)=-2.3557$. Note that we generate new function values at the two data points for each case. That is, we do not restrict all the random functions to pass through the same point. If the two data points came from a particular application, they would be fixed instead.

Appendix 2 shows the Matlab code for these examples and includes repetition of the process to extract statistical data. With 100 repetitions, the mean correlation was 0.707 with a standard deviation of 0.163 .

As the decay rate of $\theta=5$ corresponds to a fairly slowly varying function, two data points are not sparse, and we get

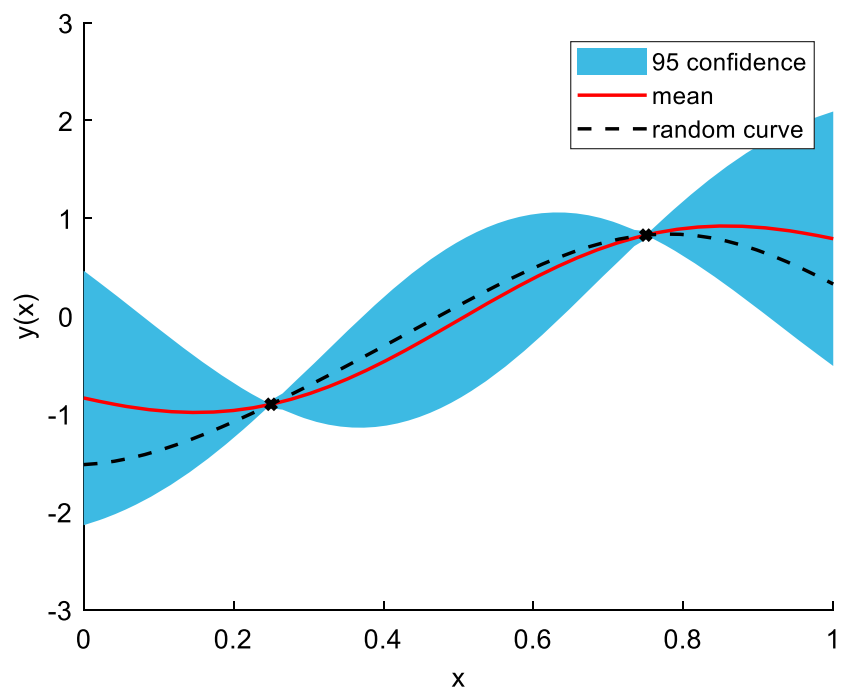

Fig. 2 One example of random data, surrogate fit, and random function from GP with $\sigma^{2}=1, \theta=5$.For this case, the correlation between error and confidence interval is 0.8554 


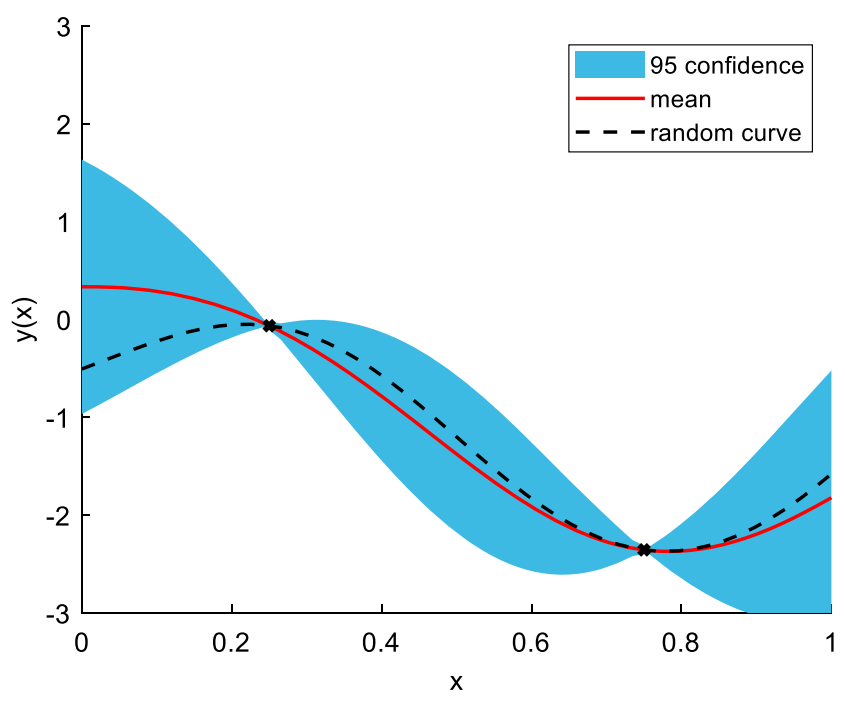

Fig. 3 A second example with $\sigma^{2}=1, \theta=5$. For this case, the correlation was 0.6608

reasonable error estimates if that true decay rate is known. When the function varies more rapidly, two points would correspond to sparse sampling. For example, Fig. 4 shows a case with $\theta=50$, where the correlation is only 0.2969 . For $\theta=50$, the mean correlation was calculated to be 0.472 and with a standard deviation of 0.158 . This is more similar to the results obtained by Zhang et al. (2019), but it is obtained for 100 random functions.

For two dimensional random function, the Python code provided in the supplementary material can be used. This code was used to generate Fig. 5.

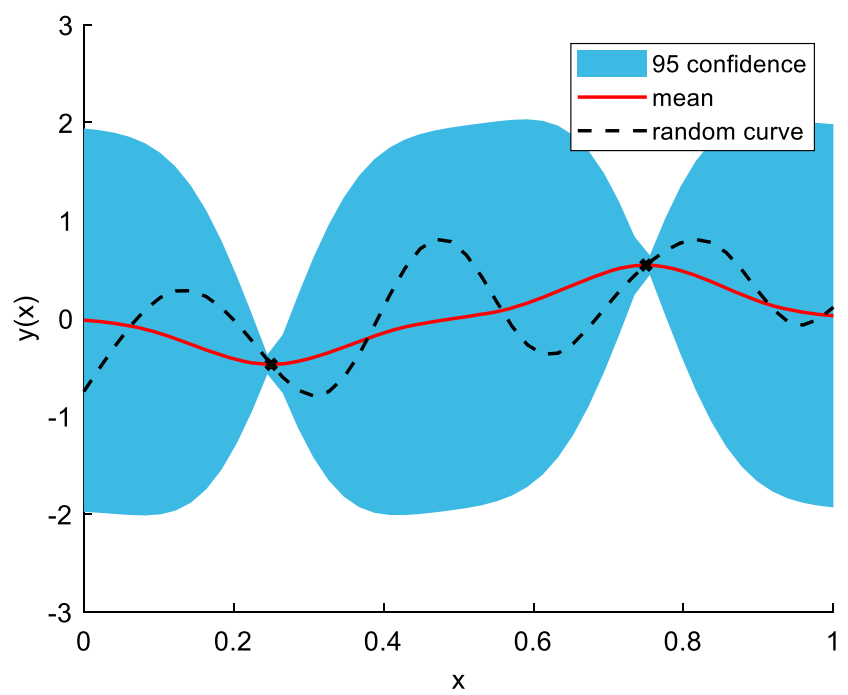

Fig. 4 An example with $\theta=50$ and a correlation of 0.2969 between the surrogate error and the $95 \%$ confidence interval width

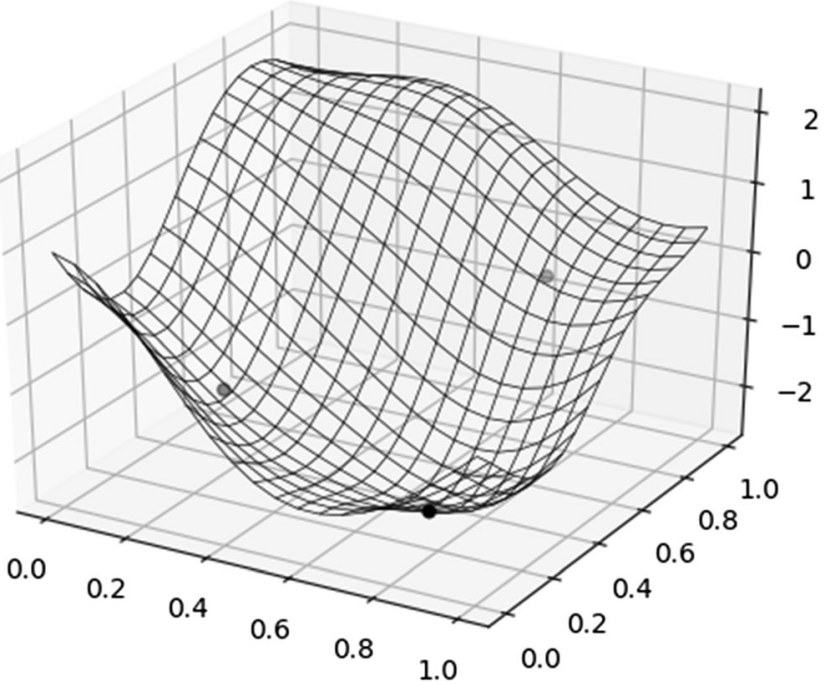

Fig. 5 2D random function interpolating 3 given points

\section{Concluding remarks}

This editorial proposes the possible use of GP for generating random functions that could be used to complement standard test functions for checking the performance of algorithms based on fitting surrogates to data. The GP is defined by spatial decay rates, and a given test function may be complemented by selecting a GP with similar decay rates. Such a complement may alert authors if their algorithms are tailored too narrowly to particular properties of their favorite test functions. The objective of this editorial is to stimulate discussion of the problem of algorithms customized to particular test functions and of alternative approaches to reduce such customization. For example, one of the challenges is that test functions are typically continuous and differentiable, a GP generalization will retain this smoothness, but many real applications have discontinuities.

\section{Compliance with ethical standards}

Conflict of interest The authors declare that they have no conflict of interest.

Replication of results The appendices provide the Matlab codes used to generate the results. 


\section{Appendix 1. Matlab code for generating} the random functions shown in Fig. 1

$n x=101 ;$ sigma=0.1; theta=5; \%This assumes that 101 points will be enough for a smooth curve $\mathrm{x}=$ linspace $(0,1, \mathrm{nx})^{\prime} ; \%$ generate function evaluation points

$m=z e r o s(n x, 1) ; \%$ mean function values at the points. Can be set to any desired mean function. $\mathrm{C}=\exp \left(-\right.$ theta*$\left.\left(\operatorname{repmat}(\mathrm{x}, 1, \mathrm{nx})-\operatorname{repmat}\left(\mathrm{x}^{\prime}, \mathrm{nx}, 1\right)\right) .{ }^{\wedge} 2\right) ; \%$ corelation matrix

randn('seed',1) \% set seed number

$\mathrm{y}=\mathrm{m}+\operatorname{sigma} * \operatorname{chol}\left(\mathrm{K}+\mathrm{nx} \mathrm{eps}^{*} \text { eye }(\mathrm{nx})\right)^{\prime} *$ randn(nx,1); \% generate a random curve

ciub=m + norminv $(0.975,0$, sigma $) ;$ cilb=m + norminv(0.025,0,sigma $) ; \% 95 \% \mathrm{Cl}$

xShaded=[x;sort(x,'descend')]; yShaded=[cilb;ciub(end:-1:1)];

patch(xShaded,yShaded,[.24 .73 .89],'EdgeColor','none'); \% plot 95\% Cl

hold on; plot(x,m, 'r-','Linewidth',1.5) \% plot the mean function

plot $\left(x, y,{ }^{\prime} k-'\right) \%$ plot the random curve

ylim([-0.3,0.3]); set(gca,'Fontsize',12);

xlabel('x','Fontsize',12); ylabel('f(x)','Fontsize',12)

\section{Appendix 2. Matlab code for section IV}


oGenerate two data points from a Gaussian Process, update the GP to othe constraint that it must pass through these two points, generate a \% random curve from this constrained GP, and use it as the true function. \%Finally, calculate the correlation between the error of the fit to the two opoints and the standard error (square root of prediction variance) \%given. Repeat many times and calculate mean and standard deviation of ocorrelation coefficient.

clear all

close all

xdata $=[0.25,0.75]^{\prime} ;$; data positions

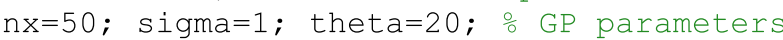

nrep=100; nplot=2; $\frac{\circ}{\circ}$ number of replications and plots

randn ('seed',1) \% set seed number

\% covariance function model

$\mathrm{K}=$ inline ('sigma^2*exp $\left(-\operatorname{theta}{ }^{*}(\operatorname{repmat}(\mathrm{x}, 1, \operatorname{size}(\mathrm{xp}, 1))-\right.$

repmat (transpose $\left.(x p), \operatorname{size}(x, 1), 1)) .{ }^{\wedge} 2\right) ', \ldots$

'x','xp', 'sigma', 'theta');

o mean function model. Here it is set to zero, but could be any function.

$\mathrm{m}=$ inline ('zeros (size $(\mathrm{x}, 1), 1)$ ', 'x');

$\mathrm{x}=$ linspace $(0,1.0, \mathrm{nx}){ }^{\prime}$; $\frac{\circ}{0}$ generate grid points

nt=length (xdata);

sigdd=K ( $x$ data, $x$ data, sigma, theta); $\frac{\circ}{\mathrm{S}}$ dd matrix

$\mathrm{m}$ upd=zeros $(\mathrm{nx}, 1) ; \mathrm{v}$ upd=zeros $(\mathrm{nx}, 1) ; \operatorname{ciub}=\operatorname{zeros}(\mathrm{nx}, 1)$;

$\mathrm{c} \overline{\mathrm{i}} \mathrm{l} \mathrm{b}=\operatorname{zeros}(\mathrm{nx}, 1)$;

for $r e p=1$ :nrep oreplication loop

\% generate data points from Gaussian process

ydata $=\operatorname{chol}($ sigdd $+n t * e p s * e y e(n t)) ' * r a n d n(n t, 1)$;

oupdate mean and covariance

sigds=K (xdata, $x$, sigma, theta) ;

sigss $=K(x, x$, sigma, theta) ;

m_upd $=m(x)+s i g d s^{\prime}$ *inv $($ sigdd $) *(y d a t a-m(x d a t a))$;

sigss_upd=sigss-sigds'*inv(sigdd)*sigds;

$\mathrm{v}$ upd=diag (sigss upd);

for $i=1: n x$

\% upper and lower bounds of 95\% confidence interval

ciub(i) $=m$ upd (i) $+\operatorname{norminv}(0.975,0, \operatorname{sqrt}(v \operatorname{upd}(i)))$;

cilb(i) $=$ m_upd $(i)+\operatorname{norminv}(0.025,0$, sqrt (v_upd(i)));

end

ogenerate random curve

yr=m_upd+chol (sigss_upd+nx*eps*eye $(\mathrm{nx})$ ) ' *randn $(\mathrm{nx}, 1)$;

ocalculate correlation of absolute error with square root of prediction variance

errab=abs (yr-m upd);

sqrtv=sqrt (v_upd);

cor $=$ corrcoef (errab, squtv);

corel $($ rep $)=\operatorname{cor}(2,1)$;

if $(r e p-n p l o t-1)<0$

\% plot $95 \%$ confidence interval

figure; hold on;

$x$ Shaded $=\left[x ; \operatorname{sort}\left(x,{ }^{\prime}\right.\right.$ descend') $] ; y$ Shaded=[cilb; ciub (end: $\left.\left.-1: 1\right)\right]$;

patch (xShaded, yShaded, [.24 .73 .89], 'EdgeColor', 'none');

plot (x,m upd,' 'r-',' Linewidth', 1.5) \% plot the mean function (most

probable prediction)

plot $\left(\mathrm{x}, \mathrm{yr}, \mathrm{k}^{--}\right.$', 'Linewidth', 1.5) 이의 the random curve

plot (xdata, ydata, 'kx', 'Linewidth', 2.5 ) data points

$\operatorname{ylim}([-3,3])$;

set (gca,' 'Fontsize', 12);

xlabel ('x','Fontsize', 12); ylabel('y(x)','Fontsize', 12)

legend('95 confidence', 'mean', 'random curve') end

end

corel

corm=mean (corel)

corstd=std (corel) 


\section{Reference}

Zhang, Y., Kim, N. H., \& Haftka, R. T. (2019). General surrogate adaptive sampling using interquartile range for design space exploration. In AIAA Scitech 2019 Forum (p. 2213).
Publisher's note Springer Nature remains neutral with regard to jurisdictional claims in published maps and institutional affiliations. 